\title{
Parenting and Adolescent's Psychological Adjustment: Toward a Systemic Approach in Parenting Research
}

\author{
Marwan Dwairy*
}

Oranim Academic College, Israel

\begin{abstract}
This article's objectives are twofold: (a) to disclose the possible distortion of the associations found in the reductionist research that prevails in many areas, in order to ensure greater caution and better understanding of such research. (b) To study the associations between family and parental factors and adolescent psychological disorders (PD) according to a systemic model that analyses eight familial factors and eleven parental factors in addition to two nominal ones: culture and the adolescents' sex. The study is based on a data collected from nine countries (1358 male and 1526 female adolescents), regarding two categories of family factors (socio-economic and connectedness) and three categories of parenting factors (control, inconsistency, and rejection) and adolescent psychological disorders (PD). To compare different levels of reductionism, four analyses of the same data were carried out, ranging from an analysis of the associations between each factor and adolescent PD (reductionist), to analysis of the associations between all the factors taken together (systemic) and adolescent PD. In addition, the systemic analysis was carried out among different groups of adolescents according to two nominal variables: culture (western and eastern) and the adolescents' sex (male female). Our results show that in a reductionist analysis most of the family and parental factors have significant associations with adolescents PD, and altogether explain 37.2\% of adolescents' PD. Most of these associations were diminished or changed in the systemic analysis and explained only $13.5 \%$ of the PD variance. The associations of the more systemic analysis changed again when two nominal factors (culture and sex) were taken into consideration. These findings indicate that reductionist analyses may lead to illusionary associations and that mixed results are an inevitable or even inherent byproduct of reductionist research.
\end{abstract}

Keywords: Parenting, adolescents, culture, collectivism, systemic.

Phenomena take place in nature and society through interactions between variables that constitute a dynamic system. In order to study a phenomenon, researchers define variables relevant to the phenomenon and develop instruments to measure these variables. Most of the studies in social science and medicine tend to be reductionist; they deal with only a few relevant variables at a time and exclude many other relevant variables. Therefore these fields of research are flooded with mixed or inconsistent results. For example, studies examining the influence of personality factors on health [1], of thinking on pain [2], debriefing on persons exposed to traumas [3, 4], viewing of violent films on violent behavior [5-7], and emotional expression on blood pressure [8] arrived at mixed and inconsistent outcomes.

\section{SYSTEMIC EXPLANATION FOR THE INCONSISTENT RESULTS}

Inconsistent results are usually attributed to differences in methodology, such as differences in the samples, tools, and data analysis. This explanation ignores a more crucial attribution- the reductionist approach in research. Inconsistent results are not necessarily caused by methodological problems; they are inherent in the reductionist approach itself: Once researchers select certain variables to study at a time and exclude other relevant

*Address correspondence to this author at the Oranim Academic College, P.O. Box 14710, Ora St. 3b, Nazerat Ellit 17000, Israel; Tel/Fax: 9724 6561541; E-mail: psy@marwandwairy.com variables, based on their theory or for practical reasons, they in fact create an artificial phenomenon. Such phenomenon is substantially different from some other artificial phenomenon, created by other researchers through their selection of a different complex of variables. We may expect both phenomena to be different from the real phenomenon in nature or society. In my article on causality and mixed results [9], I demonstrated that the association between A and $\mathrm{B}$ depends on the presence or absence of other relevant $\mathrm{C}$ variables. Thus, when reductionist researchers find a significant association between A and B, systemic research that integrates more relevant variables may change this association, because both $\mathrm{A}$ and $\mathrm{B}$ are never independent factors and have other associations with additional relevant variables (Cs) in the system. Therefore, when the association between $\mathrm{A}$ and $\mathrm{B}$ is studied in the presence of $\mathrm{Cs}$, then the variance of $B$, for instance, may be explained better by these additional factors (Cs), rather than by $A$, which had originally been perceived as having a significant association with (or explained by) these $\mathrm{C}$ factors. The difference between reductionist and systemic research can be expected to be dramatic, when some of the $\mathrm{C}$ factors have a negative correlation with A or B. In that case the positive correlation found between A and B may turn into a negative one, when $\mathrm{Cs}$ are added. Therefore, regression analyses on different groups of variables may convey to a different outcomes.

To clarify the effect of adding one variable to another, here is a simple illustration from chemistry: Simple chemistry teaches us that combining oxygen and hydrogen 
under certain conditions creates water $(\mathrm{H} 2 \mathrm{O})$. This result changes when sulphur is present, creating sulfur oxide (H2SO4), or when sodium is present, creating sodium hydroxide $(\mathrm{NaOH})$. The mutual influence between the oxygen (A) and the hydrogen (B) changes again and again in the presence or absence of tens or hundreds of bases and conditions (Cs). Every combination of bases will cause another interaction and another outcome. Thus, there can be no association between A and B that is context or systemfree, since phenomena occur within a system. This is not a case of inconsistent results vis à vis the mutual influence of oxygen and hydrogen, as it would appear to the naked eye, but rather in this case different phenomena are created as a result of different interactions. Obviously, one cannot study all the relevant variables simultaneously, but in order to better understand the system, one should attempt to study as many as possible, in order to arrive as closely as possible at the study of the real phenomenon (For more about the limitations of the reductionist approach, see [9]).

\section{INCONSISTENT RESULTS IN PARENTING RESEARCH}

This article will focus on the associations between parenting factors and children's mental health to clarify the accuracy of the results based on reductionist research. Parenting literature points to three major categories of parenting factors influencing children's mental health. (a) Baumrind $[10,11]$ identified three parenting styles, differing in the level of parental control and warmth: authoritarian, authoritative, and permissive. Authoritarian parents emphasize control and obedience and do not promote the child's autonomy. They enforce discipline through the use of punishment and expect children to obey their orders without arguing. Permissive parents enable their children to make their own decisions and regulate their own behavior. They do not behave as figures of authority and tend to be warm and supportive. The authoritative parenting style is somewhere between authoritarian and permissive modes. Children reared in this style are not completely constrained. Instead, they are allowed a reasonable degree of latitude in their behavior. Authoritative parents do enforce limits in various ways such as reasoning, verbal give-and-take, clear-cut instructions and positive reinforcement. (b) Rohner and his colleagues [12, 13] identified the parental acceptance-rejection factor. They claim that parenting styles can be placed on a continuum between acceptance (warm and affectionate) and rejection (cold, hostile, and indifferent), based on how warm they are towards their children, and (c) Dwairy [14] has recently identified the parental inconsistency factor, including three categories of inconsistency: temporal, situational, and fathermother inconsistency. Temporal inconsistency is the change in the parent behavior to the same situation from one time to another. Situational inconsistency is the change of the parent behavior from one situation (or issue) to another. Fathermother inconsistency related to the difference between father and mother behavior.

The field of parenting and children's mental health is dominated by reductionist research, each study focusing on only a few parenting factors and consequently suffering from mixed and inconsistent results. For instance, among researchers focusing on authoritarian or controlling parenting, some found that authoritarian parenting was associated with children's psychological problems [15-17], while other researchers found it associated with better psychological adjustment [18-20]. Still other researchers found minor or no significant association between authoritarian parenting and children's mental health [21]. Researchers, focusing on the association between parental rejection and psychological disorders, found inconsistent levels of effect on children's mental health: The explained variance children's psychological disorders by parental rejection differ from one study to another and ranges between $27 \%$ and $46 \%$ [22]. Correlations between parental acceptance and parental control are not consistent either and range from -.36 to -.77 (See [23]). Whatever the association found between the acceptance-rejection factor and parental control, one can assume that the shared variance between the two variables will expose the difference between reductionist analysis, analysing the association between each factor separately and children's mental health, and another less reductionist one that analyses the associations between the two parental factors and mental health together.

\section{SYSTEMIC VERSUS REDUCTIONIST RESEARCH: THE EXAMPLE OF PARENTING FACTORS}

This study is based on a worldwide data, collected from nine countries, regarding two categories of family factors and three categories of parenting factors and adolescent psychological disorders (PD). The categories of the family factors were the following: Demographic (number of siblings, father's and mother's years of education, and family economic level), and adolescent-family connectedness (emotional, financial, and economic connectedness). The categories of parenting were the following: Control (father and mother control), inconsistency (father and mother temporal inconsistency, father and mother situational inconsistency, and father-mother inconsistency), and acceptance-rejection (father and mother rejection, and father and mother acceptance). A total of nineteen familial and parenting factors were taken into account, enabling us to conduct a more systemic analysis.

To compare different levels of reductionism, four analyses of the same data were carried out, ranging from an analysis of the associations between one factor at a time and adolescent PD (reductionist), to analysis of the associations between all the factors taken together (systemic) and adolescent PD. In addition, the systemic analysis was carried out among different groups of adolescents according to two nominal variables: culture (western and eastern) and the adolescents' sex (male female).

This article's objectives are twofold: (a) to disclose the distortion of the associations found in the reductionist research that prevails in many areas in order to ensure greater caution and attentiveness to the dangers of such research. (b) To study the associations between family and parental factors and adolescent psychological disorders (PD) according to a systemic model that analyses eight familial factors and eleven parental factors in addition to two nominal ones: culture and the adolescents' sex.

The following hypotheses were tested: The associations found between family and parental factors and PD via systemic research will be substantially different from those found by means of reductionist analysis. Owing to the shared 
variance between the factors involved in this research, the variance of $P D$ explained by all the factors in a systemic analysis will be much smaller than the sum of the explained variance found in the reductionist analyses.

\section{METHOD}

\section{Sample}

Questionnaires probing family and parenting factors and psychological disorders were administered in three western countries (France, Poland, and Argentina), and six eastern countries (Kuwait, Algeria, Saudi Arabia, Bedouins in Israel, Jordan, and India), to 1358 male and 1526 female adolescents (Table 1). Their ages ranged from 15 to 17 years old, while studying in the $10^{\text {th }}, 11^{\text {th }}$, and $12^{\text {th }}$ grades $(469$, 1477, and 938 respectively). The questionnaires were administered at the schools and during a period lasting 50-60 minutes in each class. In accordance with each country's law, the consent of the school inspector and/or the parents was obtained. Participation was voluntary; however, there were no refusals. Except for the samples in India and Bedouins in Israel, all the samples included urban and rural respondents. More urban respondents were included in the eastern sample (83.0\%) than in the western one $(69.3 \%)$. The Indian sample came from middle and higher strata of society, from students studying in an English speaking school in India. The Bedouins in Israel are Arabs who live in a tribal social system [24-26].

Table 1. The Sample According to Western and Eastern Country, and Sex

\begin{tabular}{|l|c|c|c|}
\hline \multicolumn{1}{|c|}{ Culture } & Male & Female & Total \\
\hline \hline Western & 364 & 478 & 842 \\
\hline Eastern & 994 & 1048 & 2042 \\
\hline Total & $\mathbf{1 3 5 8}$ & $\mathbf{1 5 2 6}$ & $\mathbf{2 8 8 4}$ \\
\hline
\end{tabular}

Some of the family factors were collected by means of a demographic questionnaire used in previous studies $[14,21$, 27]. The respondents were asked to report the number of their siblings, the father's and mother's years of education, and to rate their family's economic level from $1=$ very low to $5=$ very high "as compared to the other students in the school." The mean in the number of siblings in the western and eastern families was 1.94 and 5.00, respectively. The mean in the number of the fathers' school years was 12.62 in the west and 12.93 in the east, suggesting that the vast majority of the parents had finished high school. The percentage of fathers with higher education was $62.6 \%$ in the west and $62.7 \%$ in the east. The percentage of mothers with higher education was $67.7 .6 \%$ in the west and $54.9 \%$ in the east. The mean of the families' economic level was 3.13 in the western sample and 3.23 in the eastern sample. These differences do not indicate the objective differences in the economical level of the western and eastern families, because the respondents were asked to compare the economic level of their family on a subjective rating scale to the other students in their own school.

\section{Instruments}

All the questionnaires were translated into the language of each country and translated back to the original language (English or Arabic). The translation was carried out by professionals who knew the two languages and the rationale underlying each instrument. Except for India, the questionnaires were administered to the respondents in their mother tongue. In India the questionnaires were administered in English in English speaking schools to Indian adolescents from the middle and upper class. Factor analyses and alpha Cronbach's coefficients indicated good validity and were reported elsewhere for each questionnaire. Details of the questionnaire are provided below:

\section{Multigenerational Interconnectedness Scale (MIS)}

This scale was developed by Gavazzi and Sabatelli [2830], and consists of three subscales, intended to assess emotional, financial, and functional connectedness between the adolescent and his/her family. For the present study, a shortened version of the Arabic MIS was used, which comprises five items each for emotional, financial, and functional connectedness (total 15 items). A principal factor analysis conducted on the present sample with a priori threefactor solution and varimax rotation showed good convergence of the items into three subscales that explained $44.6 \%$ of the variance. The alpha coefficients of the whole shortened scale were .78 and of the emotional, financial, and functional subscales were $.70, .73$, and .77 , respectively.

In order to validate the MIS across cultures two separate principal factor analyses were conducted on the western and eastern samples with a priori three-factor solution and varimax rotation. Good convergences of the items into three subscales were displayed and explained $38.18 \%$ and $47.12 \%$ of the variance in the west and east respectively indicating the validity of the scale across cultures. The alpha coefficients of the whole shortened scale were .69 among the western and .79 among the eastern sample.

\section{Dwairy's Parental Authoritarianism and Inconsistency Scale (DPAIS)}

The scale was developed by the author [14], to assess authoritarianism in conjunction with parental inconsistency. It comprises two parallel parts, each calling for the fathers' or the mothers' responses. In each part, ten parent-adolescent conflicts are addressed, such as conflicts concerning social behavior, sibling relationships, clothing, school homework, and so on. For each conflict two measures are taken: parental control and temporal parental inconsistency. The adolescents are asked to rate for each conflict the parent's (father or mother) response on a 5-point Likert scale (ranging from $5=$ controlling/punishing to $1=$ accepting/forgiving). Based on the average score the Father Control (FC) and Mother Control (MC) are assessed. The sum of FC and MC indicates the general Parental Control (PC). For each conflict the subject is asked to rate the parent's response consistency on a scale that ranges from $5=$ inconsistent and changing from time to time, to $1=$ always consistent. Here are two examples concerning conflict in connection with the father's need for help: 
Father's control item:

When I do not obey my father when he asks me for help, his response will be:

Accepting/forgiving

\section{Controlling/punishing}

$\begin{array}{lllll}1 & 2 & 3 & 4 & 5\end{array}$

Father's temporal inconsistency item:

When I do not obey my father when he asks for help, his response is usually:

Always consistent Inconsistent \& changing

$\begin{array}{lllll}1 & 2 & 3 & 4 & 5\end{array}$

Based on the average scores of the ten temporal inconsistencies, the Temporal Inconsistency of the father (FTI) and of the mother (MTI) were assessed. In addition, two other inconsistencies were assessed: a) Situational Inconsistency (SI), assessing inconsistency in the parents' responses across the conflicts or situations. The average of the absolute differences between the father's control scores in each situation and the mean of the father's control (FC) is considered as the Father Situational Inconsistency (FSI), and of the average of the absolute differences of the mother's as the Mother Situational Inconsistency (MSI). b) FatherMother Inconsistency (FMI) assessing the inconsistencies between the two parents in the same situation and is calculated by averaging the absolute differences between the rated control of the father and that of the mother for each situation.

A principal factor analysis was conducted on the 20 items concerning the fathers' responses (FAP and FTI) with varimax rotation, a priori two factors solution, and a .20 loading criterion. The two factors explained $35.7 \%$ of the variance. All items of FAP were loaded in one factor and all FTI items were loaded in the second factor. A second principal factor analysis was conducted on the 20 items concerning the mothers' responses (MAP and MTI) with varimax rotation, a priori two factors solution, and a .20 loading criterion. The two factors explained $42.8 \%$ of the variance. All items of MAP were loaded in one factor and all MTI items were loaded in the second factor. Alpha Cronbach's coefficients of FAP, FTI, MAP, and MTI were $.75, .83, .81$ and .88 , respectively, indicating a good internal consistency of the scales.

In order to validate the DPAIS across cultures, two separate factor analyses were conducted on the western and eastern samples with varimax rotation, a priori two factors solution, and a .20 loading criterion. The 20 items concerning the fathers' responses (FAP and FTI), the two factors explained $36.46 \%$ and $34.54 \%$ of the variance of the western and eastern samples respectively. In the two samples, all items of FAP were loaded in one factor and all FTI items were loaded in the second factor. As for the 20 items concerning the mothers' responses (MAP and MTI), the two factors explained $42.72 \%$ and $42.46 \%$ of the variance of the western and eastern samples respectively. In the two samples, all items of MAP were loaded in one factor and all MTI items were loaded in the second factor. Alpha Cronbach's coefficients of FAP, FTI, MAP, and MTI in the western sample were $.76, .83, .81$ and .87 , and of the eastern sample $.74, .82, .81$, and .88 respectively, indicating a good internal consistency of the scales across culture.

\section{Parental Acceptance Rejection Questionnaire PARQ}

The shortened form of PARQ includes 29 items, each referring to father and mother acceptance or rejection [13, 31]. The subjects respond to items on a 4-point Likert-like scale ranging from $4=$ almost always true to $1=$ almost never true. Sample items on the version about mothers include "my mother says nice things about me" (Acceptance), and "My mother sees me as a big nuisance" (Rejection). A principal factor analysis was conducted on the 29 items concerning the father and on the 29 items concerning the mother, with varimax rotation, a priori two factors solution, and a .20 loading criterion. The two factors regarding the items related to fathers and mothers explained $38.3 \%$ and $40.6 \%$ of the variance respectively. Three items $(7,20$, and 25$)$ in both the versions loaded low in one or more factors therefore it was decided to exclude them from the scale. The scale ended in 16 rejection and 10 acceptance items.

The mean of each group of items was considered as indicating rejection or acceptance by the father or the mother. Alpha Cronbach's coefficient of the father and mother PARQ was .75 and .77 respectively.

In order to validate the $P A R Q$ across cultures two separate factor analyses were conducted on the western and eastern samples with varimax rotation, a priori two factors solution, and a .20 loading criterion. The two factors solution regarding the 26 items related to fathers in the west and east explained $42.59 \%$ and $40.13 \%$ of the variance respectively. As for the items related to mothers in the west and east the two factors solution explained $43.70 \%$ and $43.52 \%$ of the variance respectively. All items were loaded either in the acceptance or rejection factor. Alpha Cronbach's coefficient of the father and mother PARQ in the west was .70 and .71 respectively, and in the east .75 and .79 respectively indicating the internal validity of the scale across cultures.

\section{The Psychological State Scale (PSS)}

This scale is based on a larger scale, the Psychological State Scale [32]. Since in our present study we are interested in a scale that assesses general mental health rather than making a differential diagnosis, and also for economic reasons, we chose to use only three psychological states with five items each (15 items), covering three psychological disorders: Generalized anxiety disorder (I feel fear and anxiety for no apparent reason; I feel anxious when I go to sleep; I wake up after a short sleep, and can't fall asleep again; I feel that my extremities are generally cold; and I feel anxious while I do things and afraid of what will happen next), depression (I feel sad most of the time; I do not enjoy life; I prefer to be alone away from people; I feel distressed for no reason; and I feel that I am about to cry), and conduct disorder (I do things contrary to other people's opinion; I like to do things that hurt others; using violence makes others respect me; I like to do things that bother others; and I always disobey orders). To allow a wider range of responses, subjects in the present research were asked to rate their level of endorsement of each item on a five-point scale (ranging from $5=$ always true to $1=$ not true at all). A principal factor analysis was conducted on the 15 items of PSS with a varimax rotation and a .20 loading criterion. Two factors 
where revealed: The first explains $26.6 \%$ of the variance and was loaded by all the ten items of anxiety and depression. All the five items of conduct disorder were loaded on the second factor that explains $18.5 \%$ of the variance. Four items were loaded in the two factors (Table 1). The alpha Cronbach's coefficient of PSS was calculated in our present sample and was .86, indicating good internal consistency of the scale.

In order to validate the PSS across cultures a factor analysis with a varimax rotation, a priori two factors solution, and a .20 loading criterion was done separately on the western and eastern samples. The two factors explained $42.33 \%$ of the variance in the west and $43.97 \%$ in the east and all items were loaded accordingly. The alpha Cronbach's coefficients in the west and east were .85 and .87 respectively indicating a good internal validity of the scale.

\section{RESULTS}

To understand the association between family and parental factors and adolescent PD, a linear multiple regression was conducted on four levels of reductionism. Table 2 shows the four regression analyses of these associations. The first was a reductionist analysis, conducted separately between one familial or parental factor and psychological disorders; the second was on groups of familial or parental factors and psychological disorders; the third was on all familial factors or all parental factors and psychological disorders; and the fourth analysis, the more systemic one, was conducted on all the factors together and psychological disorders. In the first and reductionist analysis, most of the familial factors and all the parental factors were associated to PD. Many of these significant associations were diminished in the fourth systemic analysis (e.g. parents' education) and many others were reduced (e.g. father rejection). The sum of the explained variance in reductionist analyses was more than twice higher $\left(\mathrm{R}^{2}=.372\right)$ than the explained variance in the systemic one $\left(\mathrm{R}^{2}=.135\right)$, indicating high shared variance between the factors.

These findings should not be considered as the last word on the subject, because the analysis did not take into account many other relevant factors, such as culture and the adolescents' sex. Taking these factors into account is expected to cause significant changes in the associations found. Therefore an additional, more systemic regression analysis was conducted on western and eastern adolescents and on male and female adolescents (Table 3).

The distinction between Western and Eastern countries was validated when we compared the level of familial connectedness. Fig. (1) indicates that adolescents in the three Western countries (France, Poland, and Argentina) were less connected to their families than adolescents in the eastern countries (Kuwait, Algeria, Saudi Arabia, Bedouins in Israel,

Table 2. Standardized Coefficients ( $\beta$ ) Between Familial and Parental Factors and Psychological Disorders According to Four Analyses Ranging from Reductionist to More Systemic Analyses

\begin{tabular}{|c|c|c|c|c|c|c|c|c|c|c|}
\hline & & & \multicolumn{2}{|c|}{$\begin{array}{l}\text { Each Factor } \\
\text { (Reductionist) }\end{array}$} & \multicolumn{2}{|c|}{$\begin{array}{l}\text { Each Group } \\
\text { of Factors }\end{array}$} & \multicolumn{2}{|c|}{$\begin{array}{c}\text { Familial and } \\
\text { Parental Factors }\end{array}$} & \multicolumn{2}{|c|}{$\begin{array}{l}\text { All Familial and Parental } \\
\text { Factors (Systemic) }\end{array}$} \\
\hline \multirow{8}{*}{$\begin{array}{c}\mathrm{F} \\
\mathrm{A} \\
\mathrm{M} \\
\mathrm{I} \\
\mathrm{L} \\
\mathrm{Y}\end{array}$} & & & $\mathrm{R}^{2}$ & $\beta$ & $\mathrm{R}^{2}$ & $\beta$ & $\mathrm{R}^{2}$ & $\beta$ & $\mathrm{R}^{2}$ & $\beta$ \\
\hline & 1 & Siblings \# & .013 & $.11 * * *$ & \multirow{4}{*}{.002} & n.s & \multirow{7}{*}{.030} & $.06 *$ & \multirow{19}{*}{.135} & n.s \\
\hline & 2 & Father education & .003 & $-.05^{*}$ & & n.s & & n.s & & n.s \\
\hline & 3 & Mother education & .000 & n.s & & n.s & & n.s & & n.s \\
\hline & 4 & Family economic & .002 & $-.05^{*}$ & & n.s & & n.s & & n.s \\
\hline & 5 & Emotional cnct & .000 & n.s & \multirow{3}{*}{.026} & $.09 * * *$ & & $.06 \%$ & & $.09 * *$ \\
\hline & 6 & Financial cnct & .003 & $-.06 * *$ & & n.s & & $-.06 *$ & & $-.05 *$ \\
\hline & 7 & Functional cnct & .019 & $-.14 * * *$ & & $-.16^{* * *}$ & & $-.15 * * *$ & & n.s \\
\hline \multirow{12}{*}{$\begin{array}{l}P \\
A \\
R \\
E \\
N \\
T \\
A \\
L\end{array}$} & 1 & Father control & .010 & $.10 * * *$ & \multirow{2}{*}{.009} & $.10 * * *$ & \multirow{11}{*}{.170} & n.s & & n.s \\
\hline & 2 & Mother control & .004 & $.06 * *$ & & n.s & & $.05 *$ & & n.s \\
\hline & 3 & Father tmp. incns. & .028 & $.17 * * *$ & & $.12 * * *$ & & $.09 * *$ & & $.08 *$ \\
\hline & 4 & Mother tmp. incns & .021 & $.15^{* * *}$ & & $.06 *$ & & n.s & & n.s \\
\hline & 5 & Father situ. incns & .003 & $.05 * *$ & .040 & n.s & & $.06 *$ & & $.06 *$ \\
\hline & 6 & Mother situ. incns & .004 & $.06 * *$ & & n.s & & n.s & & $.06 *$ \\
\hline & 7 & Father-Mother incns & .011 & $.11 * * *$ & & $.08 * * *$ & & n.s & & n.s \\
\hline & 8 & Father rejection & .100 & $.32 * * *$ & \multirow{4}{*}{.137} & $.21 * * *$ & & $.20 * * *$ & & $.16^{* * *}$ \\
\hline & 9 & Mother rejection & .084 & $.29 * * *$ & & $.13 * * *$ & & $.13 * * *$ & & $.09 *$ \\
\hline & 10 & Father acceptance & .036 & $-.19 * * *$ & & $-.08 * *$ & & $-.10 * * *$ & & $-.11 * * *$ \\
\hline & 11 & Mother acceptance & .031 & $-.18 * * *$ & & $-.05 *$ & & $-.06 *$ & & $-.06 *$ \\
\hline & & Sum $R^{2}$ & .372 & & .214 & & .200 & & & \\
\hline
\end{tabular}


Jordan, and India). The total connectedness mean of the three Western countries was lower $(\mathrm{M}=4.94, \mathrm{STD}=0.75)$ than that of the six Eastern countries $(\mathrm{M}=5.51, \mathrm{STD}=0.92)$. The difference was significant with $\mathrm{p}<0.0001$. These findings enable us to deal with our data on the basis of two major cultures that were bifurcated as Western and Eastern culture.

Table 3. Standardized Coefficients ( $\beta$ ) Between Family and Parental Factors, and Psychological Disorders Analyzed Together in One Regression Among All Subjects, and Each Western and Eastern Societies and Among Each Female and Male Adolescents

\begin{tabular}{|l|c|c|c|c|c|}
\hline & All & West & East & Female & Male \\
\hline \hline Siblings \# & n.s & n.s & n.s & $\mathbf{- . 0 7 *}$ & n.s \\
\hline Father education & n.s & n.s & n.s & n.s & n.s \\
\hline Mother education & n.s & n.s & n.s & n.s & n.s \\
\hline Family economic & n.s & n.s & n.s & n.s & n.s \\
\hline Emotional cnct & $\mathbf{. 0 9 * *}$ & $\mathbf{. 1 0 *}$ & $\mathbf{. 0 7 *}$ & $\mathbf{. 0 8 *}$ & n.s \\
\hline Financial cnct & $\mathbf{- . 0 5 *}$ & $\mathbf{- . 1 0 *}$ & n.s & n.s & n.s \\
\hline Functional cnct & n.s & $\mathbf{- . 1 4 * *}$ & n.s & $-\mathbf{. 0 9 *}$ & n.s \\
\hline Father control & n.s & $\mathbf{. 1 2 *}$ & n.s & n.s & n.s \\
\hline Mother control & n.s & n.s & n.s & n.s & n.s \\
\hline Father tmp. incns. & $\mathbf{. 0 8 *}$ & $\mathbf{. 1 2 *}$ & n.s & n.s & n.s \\
\hline Mother tmp. incns & n.s & n.s & n.s & n.s & n.s \\
\hline Father situ. incns & $\mathbf{. 0 6 *}$ & n.s & n.s & n.s & n.s \\
\hline Mother situ. incns & $\mathbf{. 0 6 *}$ & n.s & $\mathbf{. 0 7 *}$ & n.s & $\mathbf{. 1 0 *}$ \\
\hline Father-Mother incns & n.s & $\mathbf{. 1 1 *}$ & n.s & $\mathbf{. 0 8 *}$ & n.s \\
\hline Father rejection & $\mathbf{. 1 6 * * *}$ & $\mathbf{. 3 1 * * *}$ & $\mathbf{. 1 0 *}$ & $\mathbf{. 1 8 * * *}$ & $\mathbf{. 2 2 * * *}$ \\
\hline Mother rejection & $\mathbf{. 0 9 *}$ & n.s & $\mathbf{. 1 5 * *}$ & $\mathbf{. 1 8 * * *}$ & n.s \\
\hline Father acceptance & $\mathbf{- . 1 1 * * *}$ & n.s & $\mathbf{- . 1 2 * *}$ & n.s & $\mathbf{- . 1 8 * * *}$ \\
\hline Mother acceptance & $\mathbf{- . 0 6 *}$ & n.s & $\mathbf{- . 0 9 *}$ & n.s & n.s \\
\hline Sum R ${ }^{2}$ & .135 & .230 & .126 & .211 & .145 \\
\hline Significant at $\alpha<0.05, * *$ & Significant at $\alpha<0.001$, and $* * *$ Significant at & $\alpha<0.0001$. \\
\hline
\end{tabular}

The associations between all family and parental factors together and PD were dramatically influenced by the cultural variable (western and eastern cultures) and by the adolescents' sex. The vast majority of associations of the familial and parental factors were not persistent, but rather culturally or sexually dependent. The only factor that was consistently associated with PD despite the differences in culture and the adolescents' sex was the fathers' rejection. The number of siblings was associated with better mental health only among female adolescents. The associations between connectedness and PD were dependent on culture and the adolescents' sex. Emotional connectedness was associated with higher PD in all the categories, but not among male adolescents. Financial and functional connectedness was associated with better mental health, in particular among western adolescents, but not among male adolescents. The associations between parental control and PD were dependent on culture. No significant associations were found between mothers' control and PD. Fathers' control was significantly associated with PD only in western culture. The associations between parental inconsistencies and PD were dependent on culture and on the adolescents' sex. Parental acceptance and rejection associations were dependent on culture and also on the adolescents' sex.

\section{DISCUSSION}

\section{Reductionist Versus Systemic Research}

The comparison between the reductionist and systemic analyses supports our hypotheses. Most of the associations between family and parenting factors and PD seemed significant in the reductionist analysis. Many of these associations were diminished or changed in the systemic analysis. The associations of the more systemic analysis changed again when two nominal factors (culture and sex) were taken into consideration. The variance of the PD explained by the factors in the reductionist analysis was much higher than that in the more systemic analysis. These findings indicate that reductionist analyses may lead to selection-dependent associations that are in fact due to relevant excluded unseen factors, concealed behind the factors chosen to be analyzed.

Notwithstanding the possible influence of different methodologies (instruments or sampling) on the results, the comparison between the results of different levels of reductionism showed that contradictory or mixed results may be generated owing to the reductionism rather than to methodological differences. Mixed results are an inevitable or even inherent byproduct of reductionist research. Each column in Tables $\mathbf{2}$ or $\mathbf{3}$ may be considered as representing the results of different studies regarding the same group of factors, ending in inconsistent results. For instance, mothers' temporal inconsistency appears to be associated with PD in one study (the reductionist one), but not associated with PD in another (the systemic one) (Table 2). Mothers' rejection was associated with PD in two studies (of female and eastern adolescents), but not significant in two others (of male and western adolescents) (Table 3). Interestingly, when a factor that has a positive association with PD (e.g. emotional connectedness) is analyzed together with a factor that has a negative association with PD (e.g. financial connectedness), a new association can appear (between emotional connectedness and PD) in a more systemic analysis, not found by means of reductionist analysis (see Table 2 ).

The limited generalization of the reductionist approach is not new, rather it is well known among researchers. Despite that, this approach still dominates much research such as research conducted on parenting; therefore most of the results that appear to be objective and scientific are in fact state-dependent or illusory. What makes this situation dangerous is the fact that many crucial decisions are taken on the basis of these results in the fields of education, psychology, medicine, sociology, and politics.

Our results do not reject the use of reductionist research, since it is useful in identifying specific factors that are relevant to the system in order to include them later on in more systemic analyses. It is useful too in learning about the distribution of the factors and for comparing groups. As for learning about the associations and interactions between factors to understand how a system works, one should be 


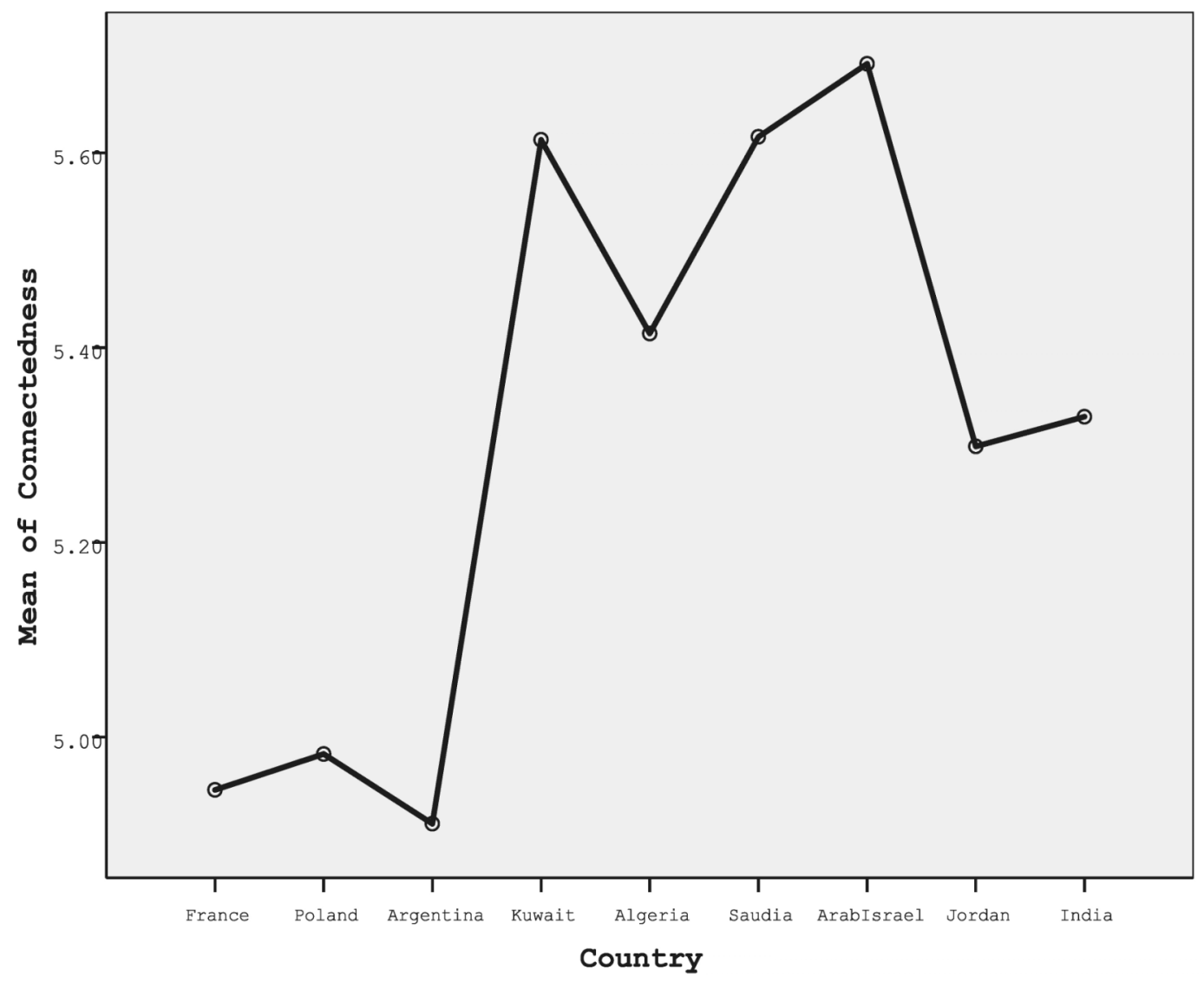

Fig. (1). Connectedness across countries.

extremely wary of reductionist research. Our results are expected to encourage researchers in the social sciences and medicine to adopt more systemic research before reaching conclusions concerning the system and before making decisions that may impact peoples' lives.

\section{Family and Parenting Factors and PD}

It appears that the numbers of siblings, parents' education, and family's economic level have no significant associations in themselves with PD, but rather via the factors of connectedness and the other parental factors. The functional connectedness, fathers' control, mothers' control, mothers' temporal inconsistency, and father-mother inconsistency also appears to be associated with PD via the other parenting factors and not in themselves. Although parental rejection was associated with PD in all the analyses in the systemic analysis, the associations were greatly reduced in the systemic analysis, indicating that the association of parental rejection with PD is shared with connectedness and parental inconsistencies.

Regardless of the culture and the adolescents' sex, the demographic factors of the family and of the parents (number of siblings, parents' education, and the family's economic level) have no associations with PD. The associations found with these factors in a reductionist analysis were misleading, because these associations were shared by other familial and parental factors and cannot be considered by themselves. Emotional connectedness was associated with higher PD and financial connectedness was associated with better mental health. Fathers' temporal inconsistency, fathers' situational inconsistency, and mothers' situational inconsistency were associated with higher PD. Father and mother rejections were associated with higher PD, and father and mother acceptance were associated with better mental health.

\section{Family and Parenting Factors and PD in Both the Western and Eastern Countries}

The associations found among all respondents regardless of culture and the adolescents' sex were different when the culture factor was taken into account. Interestingly, the variance of PD explained by familial and parental factors was higher in the west $\left(\mathrm{R}^{2}=.230\right)$ than that in the east $\left(\mathrm{R}^{2}=.126\right)$. This may be attributed to the fact that eastern children live in more extended families, where grandparents, uncles, aunts, nephews and cousins have daily relations with the children, which make the familial and parental factors within the nuclear family less important than in the west, where children are influenced mainly by their parents.

Connectedness seems to be associated with PD in the west much more than in the east. In both cultures emotional connectedness was associated with higher PD, but financial and functional connectedness was associated with better mental health only among western adolescents. Taking into account that western culture tends to be more individualistic and the western family is less connected than the eastern family $[27,33,34]$, it appears that the process of individuation taking place in the west occurs at the cost of more psychological disorders among adolescents. 
Fathers' and mothers' control was not associated with PD in the east. Fathers' control was only associated with PD in the West. To understand these cultural differences one should remember that control is considered normal in the authoritarian eastern societies and has no negative connotations [34, 35], whereas in the west, characterized as more democratic and liberal, control is considered to be a more unusual measure of parenting.

The mental health of eastern adolescents appears to be associated with parental rejection or acceptance, while the mental health of western adolescents is associated exclusively with fathers' rejection, but not with mothers' rejection or fathers' and mothers' acceptance. One possible explanation for these cultural differences may be attributed to the collective nature of the eastern cultures. In such cultures, the familial relationships are very close and expected to be warm therefore rejection or lack of acceptance in such families may hurt the children more than it would be in an individualistic culture, where the children are raised to be more independent.

Yet, it is not easy to explain why fathers' control and rejection rather than mothers' control and rejection were associated with PD, despite the fact that Western mothers are more controlling than fathers. One possible explanation is that western fathers become more involved and controlling and sometimes rejecting when the children display behavioral or psychological problems, hence the association found between fathers' control or rejection and PD in the west. Generally speaking, results shows that fathers, more than mothers, are associated with adolescents' mental health in the west, and mothers, more than fathers, are associated with mental health in the east.

As to the associations between parental inconsistencies and PD, it appears that the culture caused clear distinctions in these associations. The mental health of western adolescents was associated with fathers' temporal inconsistency and father-mother inconsistency, whereas mental health of eastern adolescents was associated with mothers' situational inconsistency. The absence of mothers' inconsistency's associations with PD exclusively in the west and the presence of the association between mothers' situational inconsistency and PD exclusively in the east may be attributed to the differences between the two cultures in the parents' roles, mentioned above.

\section{Family and Parenting Factors and PD Among Male and Female Adolescents}

When the adolescents' sex is taken into account, we see significant changes in the associations found between familial and parental factors and adolescents' PD. Generally speaking, familial and parental factors explain more variance of female $\left(\mathrm{R}^{2}=.211\right)$ than male $\left(\mathrm{R}^{2}=.145\right) \quad \mathrm{PD}$. These differences may be attributed to the wider range of influences males experience, minimizing the familial and parental influences on them and keeping these influences more dominant among females. These differences between male and female adolescents may explain why PD of males was not associated with family connectedness while the PD of females was associated with emotional and functional connectedness.
Fathers' acceptance and fathers' rejection were associated with male PD, whereas the female PD was associated exclusively with fathers' and mothers' rejection. In other words, PD of male adolescents is sensitive to the fathers' reactions (rejection or acceptance), whereas the PD of female adolescents is sensitive to rejection (either the fathers' or the mothers' rejection). As to the associations between parental inconsistencies and adolescents' PD, it seems that these associations are minor among males and females.

Although psychology and education greatly emphasize the role parents play in their children's mental health, our results show that the variance of PD explained by family and parental factors vary from approximately $13 \%$ to $23 \%$, depending on the population focused on. This finding is disappointing for those who believe that parents have more effective role, but not very different from recent metaanalytical research, showing that the variance of $\mathrm{PD}$ explained by parenting is very low [36-38]. It appears that children's psychological adjustment is associated with many other factors such as peers, siblings, school, culture, and genetics. Of course, parents still have a tremendous influence on what their children will be exposed to or how they cope with most of these factors. Therefore the actual effect of the parents' behavior and of the family in general on the children is assumed to be much higher than the explained variance of mental health found in this study.

The large and cross-cultural sample and the large number of variables measured are among the major strengths of this research, yet it was based exclusively on self-reported data, collected from adolescents. There is a need to conduct more systemic research using other instruments, such as selfreports collected from parents and observation of the children and the parents. Although our research tends to be systemic, yet it excludes other factors relevant to children's mental health. Future research needs to be more inclusive and analyze additional familial factors, such as siblings' behavior and attitudes, social factors such as peers' and teachers' behavior and attitudes, and genetic factors together with parental factors.

\section{ACKNOWLEDGEMENTS}

I wish to thank my colleague Mustafa Achoui who administered the scales and encoded the data in Saudi Arabia, Algeria, and Kuwait, and to thank Anna Filus (Poland), Neharika Vohra (India), Martina Casullo (Argentina), Parissa Rezvan Nia (France), Huda Nijm (Jordan, and Lana Shhadi (Arab in Israel), for their help in translating and administering the questionnaires and encoding the data of their countries.

\section{REFERENCES}

[1] Smith TW, Gallo LC. Personality trait as risk factors for physical illness. In: Baum AW, Revenson TA, Singer JE, Eds. Handbook of health psychology. New York: Lawrence Erlbaum Associates, Inc. 2001.

[2] Turk DC. Physiological and psychological bases of pain. In: Baum AW, Revenson TA, Singer JE, Eds. Handbook of health psychology. New York: Lawrence Erlbaum Associates, Inc. 2001.

[3] Meichenbaum, DA. clinical handbook: practical therapist manual for assessing and treating adults with post-traumatic stress disorder (PTSD). Ontario, Canada: Institute Press 2001.

[4] Orner RJ, Schnyder U. Reconstructing early intervention after trauma. Oxford: Oxford University Press 2003. 
[5] Bandura A. Aggression: a social learning analysis. Englewood Cliffs, NJ: Prentice Hall 1973.

[6] Feshbach S, Singer RD. Television and aggression. San Francisco: Jossy-Bass 1971.

[7] Feshbach S, Tangney J. Television viewing and aggression: Some alternative perspectives. Perspect Psychol Sci 2008; 3 (5): 387-9.

[8] Butler EA, Lee TL, Gross JJ. Does expressing your emotions raise or lower your blood pressure?. J Cross Cult Psychol 2009; 40(3): 510-7.

[9] Dwairy M. Causality and the phenomenon of contradictory outcomes in research. Lin Chuang Yi Xue Za Zhi 2006; 5: 272-82 [November 2006] Available from http://www.cjmed.net/news Content.aspx?id $=670$

[10] Baumrind D. Effects of authoritative parental control on child behavior. Child Dev 1966; 37: 887-907.

[11] Baumrind D. The influence of parenting style on adolescent competence and substance use. J Early Adolesc 1991; 11: 56-95.

[12] Rohner RP. Introduction to PARTheory studies of intimate partner relationships. Paper presented at First International Congress on Interpersonal Acceptance and Rejection, Istanbul, Turkey, June 2224, 2006.

[13] Rohner RP, Khaleque A. Eds. Handbook of parental acceptance and rejection, $4^{\text {th }}$ ed. Storrs CT: Rohner Research Publications 2005.

[14] Dwairy M. Parental inconsistency versus parental authoritarianism: Association with symptoms of psychological disorders. J Youth Adolesc 2007; [Retrieved: 9 October, 2009]. Available from http://springerlink.com/content/jjw15q40q771t047/fulltext.pdf?page $=1$

[15] Kochanska G, Knaack A. Effortful control as a personality characteristic of young children: Antecedents, correlates, and consequences. J Pers 2003; 71: 1087-112.

[16] Silverman IW, Ragusa DM. Child and maternal correlates of impulse control in 24-month-old children. Genet Soc Gen Psychol Monogr 1990; 116: 437-73.

[17] Stansbury K, Zimmermann LK. Relations among child language skills, maternal socialization of emotion regulation and child behavior problems. Child Psychiatr Hum Dev 1999; 30: 121-42.

[18] Belsky J, Rha JH, Park SY. Exploring reciprocal parent and child effects in US and Korean samples. Int J Behav Dev 2000; 24: 33847.

[19] Eiden RD, Leonard KE, Morrisey S. Parental alcoholism and toddler noncompliance. Alcohol Clin Exp Res 2001; 25: 1621-33.

[20] Feldman R, Klein PS. Toddlers' self-regulated compliance to mothers, caregivers, and fathers: Implications for theories of socialization. Dev Psychol 2003; 39: 680-92.

[21] Dwairy M, Achoui M, Abouserie R, Farah A. Parenting styles, individuation, and mental health of Arab adolescents: A third crossregional research study. J Cross Cult Psychol 2006; 37(3): 262-72.

[22] Kim E, Cain K, McCubbin M. Maternal and paternal parenting, acculturation, and young adolescents' psychological adjustment in
Korean American families. J Child Adolesc Psychiatr Nurs 2006; 19(3): 112-29.

[23] Soenens B. "I will love you if you do as I say" How psychological controlling parenting undermines parent-child acceptance. International Society for Interpersonal acceptance and Rejection (Newsletter) September 2007; Vol. 1(3): pp. 1-3.

[24] Alkernawi A. Ethno-psychiatry in the Bedouin Arab society in the Negev [in Hebrew]. Tel Aviv, Israel: Hakebutz Hameo'had 2000.

[25] Alkernawi A, Graham JR. Spirit possession and exorcism in the treatment of a Bedouin-Arabic psychiatric patient. Clin Soc Work J 1997; 25: 211-22.

[26] Cwikel J, Barak N. The health and welfare of Bedouin Arab women in the Negev [in Hebrew]. Negev, Israel: Center for Bedouin Studies and Development 2002.

[27] Dwairy M, Achoui M, Abouserie R, Farah A. Adolescent-family connectedness among Arabs: a second cross-regional research study. J Cross Cult Psychol 2006; 37(3): 248-61.

[28] Gavazzi SM, Sabatelli RM. Assessing levels of individuation through multigenerational interconnectedness. Paper presented at the National Council of Family Relations $49^{\text {th }}$ Annual Conference, Atlanta 1987.

[29] Gavazzi SM, Sabatelli RM. Multigenerational interconnectedness and family involvement: assessing levels of individuation in adolescence and early adulthood. Paper presented at the National Council of Family Relations $50^{\text {th }}$ Annual Conference, Philadelphia 1988.

[30] Gavazzi SM, Sabatelli RM. Family system dynamics, the individuation process, and psychosocial development. J Adolesc Res 1990; 5(4): 500-19.

[31] Rohner RP, Khaleque A. Reliability and validity of parental control scale: a meta-analysis of cross-cultural and intercultural studies. J Cross Cult Psychol 2003; 34(6): 643-9.

[32] Hamuda MA, Imam IA. The Psychological State Scale for adolescents and adults. Cairo, Egypt: Dar El Fekr El Arabi 1996. (in Arabic).

[33] Dwairy M. Parenting styles and psychological adjustment of Arab adolescents. Transcult Psychiatr 2004; 41(2): 233-52.

[34] Kagitcibasi C. Family, self, and human development across cultures. London: Lawrence Erlbaum Associates, Publishers 2007.

[35] Dwairy M. Counseling and psychotherapy with arabs and muslims: a culturally sensitive approach. New York: Teachers College Press, Columbia University 2006.

[36] McLeod BD, Weisz JR, Wood JJ. Examining the association between parenting and childhood depression: a meta-analysis. Clin Psychol Rev 2007; 27(8): 986-1003.

[37] McLeod BD, Wood JJ, Weisz JR. Examining the association between parenting and childhood anxiety: a meta-analysis. Clin Psychol Rev 2007; 27: 155-72.

[38] Rothbaum F, Weisz JR. Parental caregiving and child externalizing behaviour in nonclinical sample: a meta-analysis. Psychol Bull 1994; 116: 55-74. 